\title{
Crumpling Effects and Specific Absorption Rates of Flexible AMC Integrated Antennas
}

Sima Noghanian

University of North Dakota, sima.noghanian@engr.und.edu

Ala Alemaryeen

\section{How does access to this work benefit you? Let us know!}

Follow this and additional works at: https://commons.und.edu/ee-fac

Part of the Electronic Devices and Semiconductor Manufacturing Commons

\section{Recommended Citation}

Sima Noghanian and Ala Alemaryeen. "Crumpling Effects and Specific Absorption Rates of Flexible AMC Integrated Antennas" (2018). Electrical Engineering Faculty Publications. 8.

https://commons.und.edu/ee-fac/8

This Article is brought to you for free and open access by the Department of Electrical Engineering at UND Scholarly Commons. It has been accepted for inclusion in Electrical Engineering Faculty Publications by an authorized administrator of UND Scholarly Commons. For more information, please contact und.commons@library.und.edu. 


\title{
Crumpling effects and specific absorption rates of flexible AMC integrated antennas
}

ISSN 1751-8725

Received on 27 th July 2017 Revised 17th October 2017 Accepted on 6th November 2017 doi: 10.1049/iet-map.2017.0652 www.ietdl.org

\author{
Ala Alemaryeen ${ }^{1}$, Sima Noghanian ${ }^{1}$ \\ ${ }^{1}$ Department of Electrical Engineering, University of North Dakota, Grand Forks, North Dakota, USA \\ 凶E-mail: ala.alemaryeen@und.edu
}

\begin{abstract}
This study presents the design of a wearable textile-based monopole antenna integrated with a flexible artificial magnetic conductor (AMC). The proposed design covers the industrial, scientific, and medical $2.45 \mathrm{GHz}$ band. An AMC reflector is utilised to isolate the human body from undesired electromagnetic radiation and to improve the antenna radiation characteristics. A monopole antenna was backed with an AMC reflector and tested in free space and on human body models. The inclusion of an AMC reflector increases the front-to-back ratio and antenna gain in free space. On the human body models, the AMC antenna provides stable performance and a reduction in specific absorption rate levels compared with using the conventional monopole antenna. Moreover, the performance of the antenna with and without the AMC reflector under different crumpling conditions has been studied and presented. The effect of crumpling on reflection phase characteristics of the AMC reflector has been analysed as well.
\end{abstract}

\section{Introduction}

Wearable communication is a fast growing field of applicationoriented research due to its potential applications such as in healthcare, military, and public safety applications [1-8]. One of the main challenges for implementing wearable communication systems is the degrading effect of the human body on antenna performance. The human body can absorb a large amount of radiated electromagnetic power from the antenna due to the lossy and dispersive nature of the body tissues. Therefore, radiation degradation and frequency-detuning problems arise when antennas operate in close proximity to the human body $[4,5]$. In addition, the body-absorbed electromagnetic power may cause adverse biological effects [6].

In the literature, researchers have shown that an artificial magnetic conductor (AMC) and electromagnetic band-gap (EBG) structures may isolate the antenna from the ambient environment, thereby avoiding the deterioration of the communication range and the adverse biological effects. In [7], a miniaturised slotted Jerusalem Cross AMC ground plane was designed to minimise the antenna's impedance mismatch caused by the high permittivity of human tissues. In [8], an inkjet-printed EBG array on a paper substrate was designed for gain enhancement of a microstrip monopole antenna. A reduction in the back radiation toward the human body was achieved due to the use of the AMC structure in [2].

In [9], we showed that an AMC-based antenna provides improved gain and the front-to-back ratio (FBR) in addition to good matching within the industrial, scientific, and medical (ISM) $2.45 \mathrm{GHz}$ band. In practical situations, for wearable antennas integrated on textile or flexible materials for on-body communication applications, the antenna will be subject to several shape distortion due to the uncertain form of the material surface during the wearer's daily activities. Wearable antennas' performance under crumpling conditions has been studied by many researchers [10-12]. We investigated the effect of bending on the proposed antenna in [9]. However, the antenna shape distortion will be more complex in practical situations.

The focus of this paper is to study the effect of crumpling on a textile coplanar-waveguide (CPW) fed monopole antenna integrated with a flexible AMC structure, in terms of input matching and radiation characteristics. In addition, the effects of crumpling on the reflection phase characteristics of the AMC reflector are examined. Finally, this study explores frequency detuning, radiation characteristics, and specific absorption rate (SAR) assessment. A three-layered planer human body model is first considered to quantify the effect of the human body on the operation of the proposed antennas, then a voxel body model is presented. Modelling and numerical analysis of the entire work were carried out using full-wave electromagnetic simulation software, CST Microwave Studio (MWS), which is based on finite integration technique [13].

The paper is organised as follows: Section 2 presents design and configuration of the monopole antenna and AMC reflector. Section 3 presents performance evaluation for monopole and AMC antennas in terms of the reflection coefficient and radiation characteristics. Section 4 reports crumpling analysis of the monopole antenna in two perpendicular planes $(E$-plane and $H$ plane) of the antenna. Section 5 presents a discussion on the challenges of AMC antenna crumpling and crumpling analysis in the $E$-plane. Section 6 presents a performance evaluation of monopole and AMC antennas in the presence of a human body model, and Section 7 draws conclusions.

\section{Design and configuration}

\subsection{Design of monopole antenna}

The monopole antenna configuration was designed based on the proposed design in [14] and is optimised to operate in the ISM $2.45 \mathrm{GHz}$ band. The radiating element and the CPW feeding line were printed on the same side of a Pellon fabric substrate with a thickness of $3.6 \mathrm{~mm}$, dielectric constant $\varepsilon_{\mathrm{r}}=1.08$, and loss tangent $\tan \delta=0.008$. Fig. 1 depicts the dimension details and the fabricated prototype of the monopole antenna. It is worth noting that the separation between the two arms $\left(w_{3}\right)$ in addition to the slot width $\left(w_{2}\right)$ were selected to achieve the least reflection coefficient $\left(S_{11}<-10 \mathrm{~dB}\right)$. Pellon fabric was chosen as the antenna's substrate since it exhibits a low profile and flexible characteristics, which enable stacking multiple layers to control the thickness of the substrate while the antenna is conformal to the user's body [15].

\subsection{Design of AMC reflector}

The AMC reflector was designed by reflection phase characterisation. The AMC reflector acts as a perfect magnetic conductor in a specific frequency band in which it provides in phase reflection characteristics. The proposed monopole antenna 


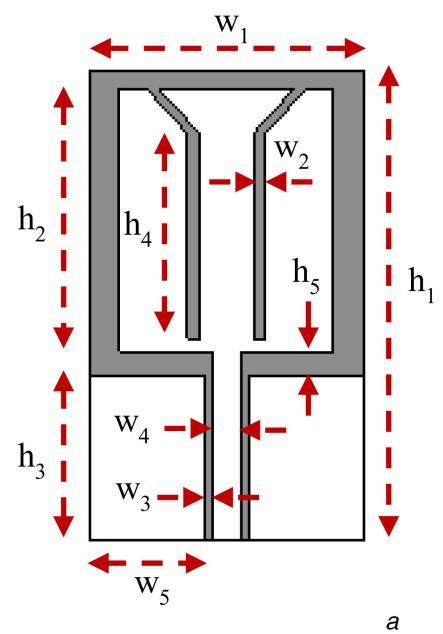

\begin{tabular}{cc}
\hline $\mathrm{h}_{1}$ & 57.00 \\
$\mathrm{~h}_{2}$ & 32.00 \\
$\mathrm{~h}_{3}$ & 20.00 \\
$\mathrm{~h}_{4}$ & 25.00 \\
$\mathrm{~h}_{5}$ & 3.00 \\
$\mathrm{w}_{1}$ & 32.10 \\
$\mathrm{w}_{2}$ & 1.00 \\
$\mathrm{w}_{3}$ & 0.30 \\
$\mathrm{w}_{4}$ & 3.00 \\
$\mathrm{w}_{5}$ & 14.00 \\
\hline
\end{tabular}

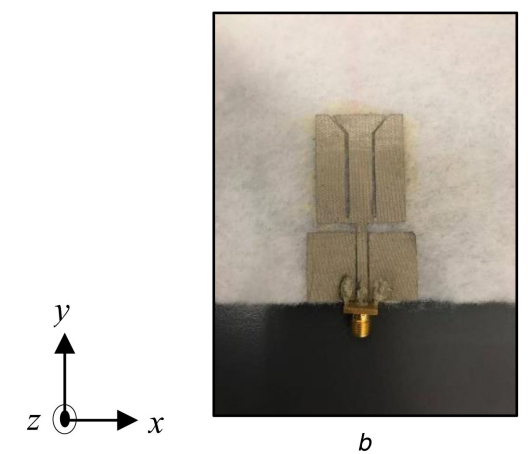

Fig. 1 Configuration of $C P W$-fed monopole antenna (a) Structure and dimensions (in mm), (b) Fabricated prototype

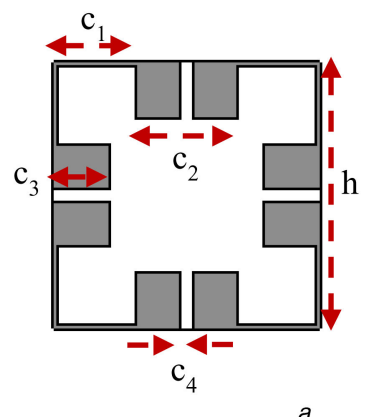

\begin{tabular}{cc}
\hline $\mathrm{c}_{1}$ & 9.00 \\
$\mathrm{c}_{2}$ & 12.00 \\
$\mathrm{c}_{3}$ & 5.50 \\
$\mathrm{c}_{4}$ & 1.30 \\
$\mathrm{~h}$ & 31.00 \\
\hline
\end{tabular}
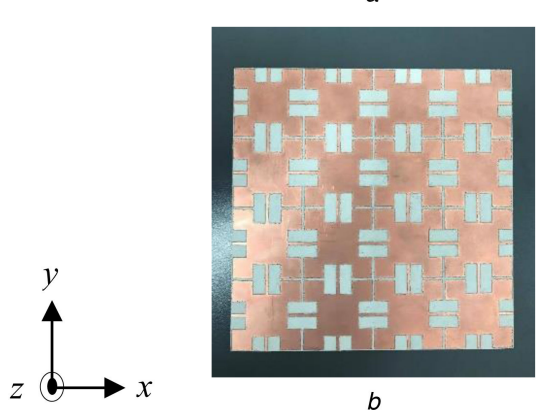

Fig. 2 Configuration of AMC reflector

(a) Structure and dimensions (in mm), (b) Fabricated prototype

was placed on a symmetric $4 \times 4$ AMC array measuring $124 \mathrm{~mm} \times$ $124 \mathrm{~mm}$. The AMC unit cell measures $31 \mathrm{~mm} \times 31 \mathrm{~mm}$, and it was printed on a $1.52 \mathrm{~mm}$ thick RO3003 flexible material with $\varepsilon_{\mathrm{r}}=3$ and $\tan \delta=0.0013$. Fig. 2 shows the configuration along with the dimension details of the proposed AMC unit cell and fabricated AMC array. The ultimate goal is to integrate AMC structures with textile antennas. Therefore, ideally, the AMC layer should also be made from a textile material. However, we found the AMC fabrication accuracy is generally low for textiles. For proof of concept, we chose to use a flexible substrate instead of textilebased AMC structures. The AMC reflection phase characterisation
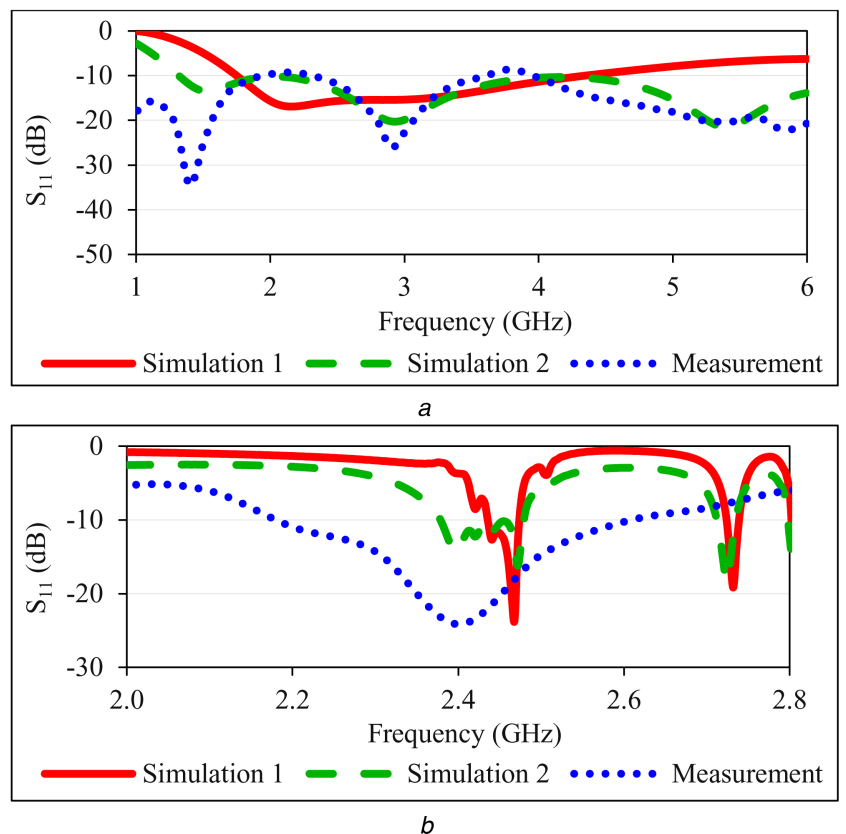

Fig. $3 S_{11}$ of wearable antennas

(a) Monopole antenna, (b) AMC antenna

procedure follows the same methodology applied in [16]. To find the AMC in the phase band, a single cell with periodic boundary conditions in the $X$ - and $Y$-directions was simulated in CST MWS. In the proposed cell, the exact point of the zero reflection phase is located at $2.45 \mathrm{GHz}$ with a narrow bandwidth of $138 \mathrm{MHz}(2.34$ $2.48 \mathrm{GHz}$ ) within $\pm 90^{\circ}$ phase values.

\section{Performance of wearable antennas on flat surface}

To study the effect of AMC proximity on impedance matching, reflection coefficient $\left(S_{11}\right)$ was simulated and measured with and without the AMC reflector as shown in Fig. 3. $S_{11}$ measurements were carried out using an Agilent E5071C Network Analyser. Discrepancies between the first set of simulations (simulation 1) and measurements are observed and may be attributed due to errors during simulation, fabrication, and measurement phases of the study. Despite the fact that the measured results confirm our design approach for the wearable antennas operating within the ISM 2.45 $\mathrm{GHz}$ band, the proposed wearable antennas do not provide the expected performance in terms of matching characteristics, therefore we started looking for the error sources in our investigation. The most potential error source we found is the error in the assumption of material properties used during the simulation phase. In the first set of simulations (simulation 1), for simplicity, we used copper of $5.8 \times 10^{7} \mathrm{~S} / \mathrm{m}$ conductivity as the conductive material in monopole antenna simulation. However, the conductivity of the electro-textile materials is different from that of a good conductor such as copper. To validate this conclusion, a parametric study on the conductivity of the electro-textile material was carried out based on simulations. Good agreement with the measurement results was obtained with the assumption of $500 \mathrm{~S} / \mathrm{m}$ as the conductivity of the electro-textile material (see simulation 2).

Many challenges were faced during the measurement phase due to the stiffness of the vector network analyser cables and SubMiniature version A (SMA) connectors and the flexibility of the textile material. We could not firmly fix the antenna and we noticed a large variation in the resonances and $S_{11}$ level when we changed the position of the antenna and cables during the setup of the test. Also, a large variation in $S_{11}$ with the pressure on the antenna connector while moving the cable was confirmed. Some problems in terms of misalignment of the monopole antenna on the AMC structure and air gap between the monopole antenna and AMC structure were also faced. The impact of a thin air layer in 


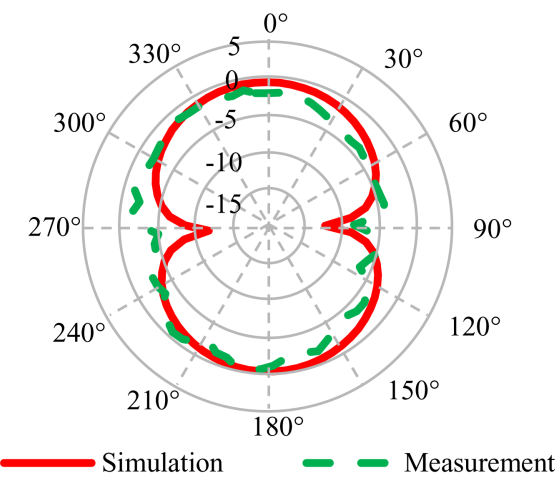

a

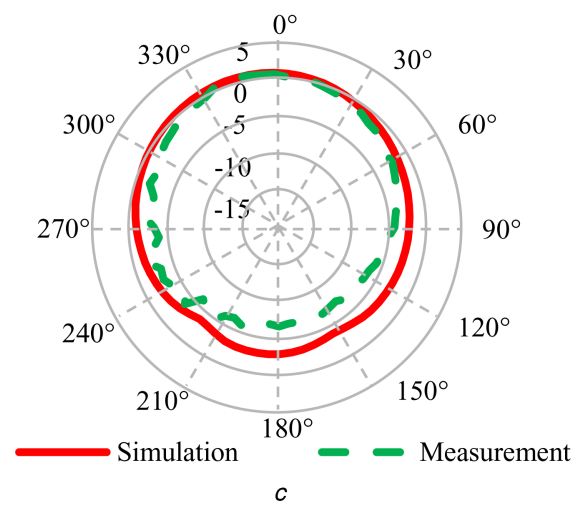

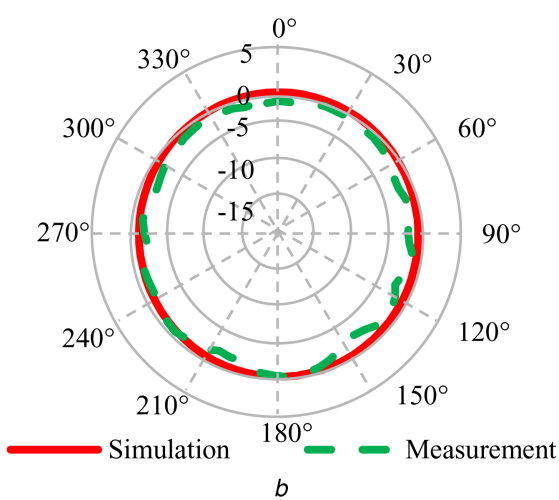

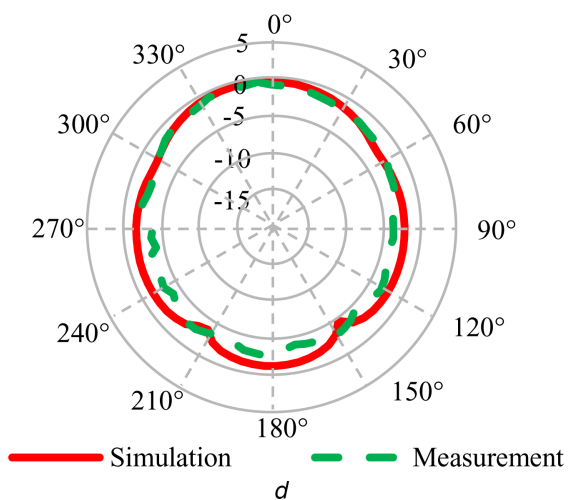

Fig. 4 Normalised radiation patterns in free space at $2.45 \mathrm{GHz}$ based on simulation and measurement results for (a) Monopole antenna (E-plane), (b) Monopole antenna ( $H$-plane), (c) AMC antenna ( $E$-plane), (d) AMC antenna ( $H$-plane)

the proposed AMC antenna, representing a layer between the textile antenna and AMC structure, was studied via electromagnetic simulation. The results showed degradation in the reflection coefficient of the antenna, and a variation of the resonance frequency and impedance bandwidth.

Simulation 2 of monopole antenna yields a wide $-10 \mathrm{~dB}$ impedance bandwidth of $4.88 \mathrm{GHz}(1.32-6.20 \mathrm{GHz})$ with a good impedance matching at $2.45 \mathrm{GHz}\left(S_{11}=-12.82 \mathrm{~dB}\right)$. Measurement result shows a good agreement with simulation 2 result within the ISM $2.45 \mathrm{GHz}$ band with a $-10 \mathrm{~dB}$ impedance bandwidth of 1.40 $\mathrm{GHz}(2.3-3.7 \mathrm{GHz})$. On the other hand, the measurement result of the AMC antenna shows a wider $-10 \mathrm{~dB}$ impedance bandwidth of $400 \mathrm{MHz}(2.2-2.6 \mathrm{GHz})$ compared with simulation 2 results $(2.38-$ $2.48 \mathrm{GHz}$ ). It is worth mentioning that the reported simulation results of the rest of simulations in this study are based on using a conductive material of $5.8 \times 10^{7} \mathrm{~S} / \mathrm{m}$ conductivity.

The effectiveness and usefulness of the AMC reflector can be assessed by comparing antenna radiation characteristics in the presence and absence of an AMC reflector. Far-field $E$-plane ( $Y Z$ plane) and $H$-plane ( $X Z$-plane) radiation patterns in free space at $2.45 \mathrm{GHz}$ are presented in Fig. 4; based on simulation and measurement results. The three-dimensional radiation pattern is quasi-hemispherical when the AMC reflector is integrated, while the original pattern is close to omnidirectional radiation. This shows an increase in the FBR and antenna directivity, achieved by the in-phase reflection of the AMC reflector. The measured antenna gain was improved from $2.60 \mathrm{dBi}$ for the monopole antenna to $4.60 \mathrm{dBi}$ for the monopole on the $\mathrm{AMC}$ reflector.

\section{Performance of monopole antenna under crumpling conditions}

In wearable applications, many types of crumpling may take place. Four typical forms of crumpling were selected as the main investigation targets in two perpendicular planes of the antenna, namely the $Y Z$ ( $E$-plane) and $X Z$ ( $H$-plane) planes. The dimensions of the antenna in each crumpling case are described in Fig. 5 for antenna crumpling in $E$ - and $H$-plane directions. On the human body, cases 1 and 2 are typical for general clothing distortion most likely to appear when the antenna is placed on the chest or the back of the wearer. Crumpling in cases 3 and 4 is likely to be observed near the knee or the elbow. The crumpling profile is defined by crumple depth $(N)$ and crumple period $(M)$. A central part of the antenna $(0.5 \mathrm{~mm})$ is kept flat for the feeding requirements imposed by the simulation program when the antenna is crumpled in the $E$ plane direction. On the other hand, antenna crumpling in the $\mathrm{H}$ plane starts from the centre of the feeding line.

Reflection coefficient measurements were carried out for crumpling cases 1 and 2 in both crumpling directions as shown in Fig. 6. In general, a good agreement between simulated and measured $S_{11}$ of the crumpled monopole antenna is achieved. In addition, a summary of the performance of all studied crumpling cases, based on simulations, is given in Table 1 . In the case of $E$ plane crumpling, stable input impedance matching within the ISM $2.45 \mathrm{GHz}$ band and a slight upward shift in the resonance frequency $\left(f_{\mathrm{r}}\right)$ were observed under different crumpling conditions.

It is worth mentioning that although the antenna is bent almost $90^{\circ}$ in case 4 , which is the most severe crumpling scenario, the $-10 \mathrm{~dB}$ impedance bandwidth is slightly reduced compared with that obtained when the antenna is flat, with $f_{\mathrm{r}}$ shifted upward by 58 MHz. Significant changes in $S_{11}$ are observed due to antenna crumpling in the $H$-plane. The $-10 \mathrm{~dB}$ impedance bandwidth is increased due to crumpling in cases 1,2 , and 3 , with the maximum bandwidth obtained in case $2(1.67-5.65 \mathrm{GHz})$ as compared with the flat antenna $(1.76-4.35 \mathrm{GHz})$. In contrast, the $-10 \mathrm{~dB}$ impedance bandwidth is halved due to crumpling in case 4 . All crumpling cases studied in $E$ - and $H$-planes successfully yield a wide $-10 \mathrm{~dB}$ impedance bandwidth that covers the ISM $2.45 \mathrm{GHz}$ band of interest.

Antenna radiation characteristics in free space at $2.45 \mathrm{GHz}$ under crumpling conditions in the $E$ - and $H$-planes are summarised in Table 1 based on simulation results. Antenna crumpling in the $E$ plane causes less distortion in radiation characteristics compared with crumpling in the $H$-plane. The most significant degradation in antenna gain and total efficiency is observed in the crumpling of case 4 along the $H$-plane. Crumpling in case 4 in the $H$-plane direction shows the highest amount of ground plane excitation. In addition, the radiation pattern measurement of the crumpled 


\begin{tabular}{c|cccccc}
\hline & & Antenna Width $(\mathrm{mm})$ & Antenna Length $(\mathrm{mm})$ & $N(\mathrm{~mm})$ & $M(\mathrm{~mm})$ & Crumpling Profile \\
\cline { 2 - 6 } & Flat & 32.10 & 57.00 & - & - \\
\hline \multirow{5}{*}{ E-plane } & Case 1 & - & 54.37 & 6.00 & 31.00 \\
Bending & Case 2 & - & 50.25 & 6.00 & 24.00 \\
& Case 3 & - & 44.92 & 11.00 & 31.00 \\
& Case 4 & - & 40.40 & 35.00 & 60.00 \\
\hline \multirow{5}{*}{ H-plane } & Case 1 & 30.00 & - & 6.00 & 31.00 \\
& Case 2 & 31.00 & - & 6.00 & 24.00 \\
& Case 3 & 27.13 & - & 11.00 & 31.00 & \\
& Case 4 & 25.70 & - & 35.00 & 60.00 & \\
\hline
\end{tabular}

Fig. 5 Dimensions of the crumpled monopole antenna
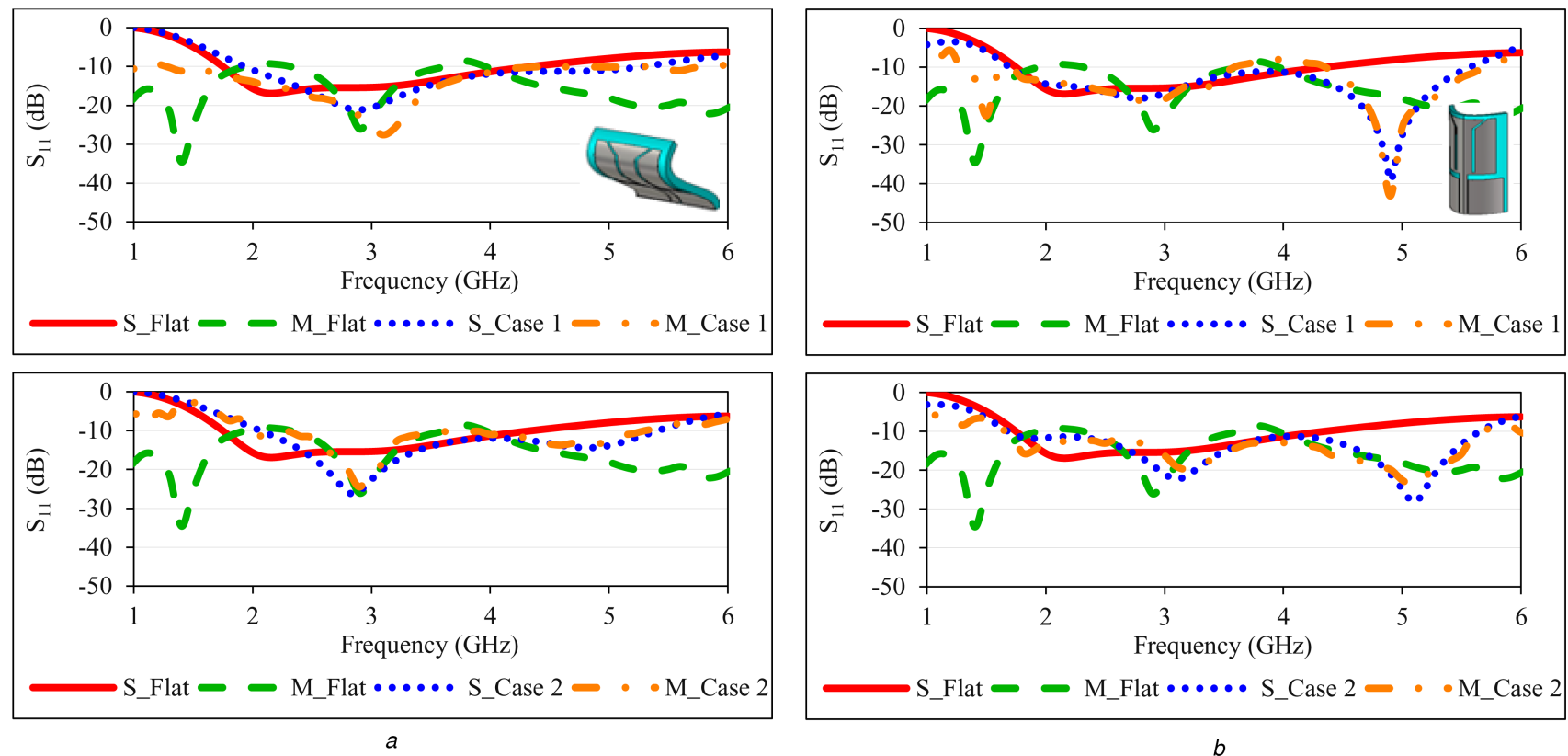

Fig. $6 S_{11}$ of crumpled monopole antenna based on simulation (S) and measurement (M) results for case 1 (top) and case 2 (bottom) in (a) E-plane direction, (b) $H$-plane direction

Table 1 Performance summary at $2.45 \mathrm{GHz}$ for monopole antenna crumpled in E-plane and $\mathrm{H}$-plane directions

\begin{tabular}{|c|c|c|c|c|c|c|}
\hline Crumpling plane & Parameter & Flat & Case 1 & Case 2 & Case 3 & Case 4 \\
\hline & $f_{\mathrm{l}}, \mathrm{GHz}$ & 1.75 & 1.92 & 2.08 & 2.32 & 1.91 \\
\hline \multirow[t]{6}{*}{ E-plane } & $f_{\mathrm{h}}, \mathrm{GHz}$ & 4.33 & 4.08 & 5.04 & 5.54 & 3.83 \\
\hline & $S_{11}, d B$ & -16.92 & -16.06 & -15.83 & -11.42 & -16.58 \\
\hline & gain, $\mathrm{dBi}$ & 2.45 & 2.45 & 2.32 & 2.21 & 2.32 \\
\hline & directivity, dBi & 2.67 & 2.66 & 2.50 & 2.58 & 2.50 \\
\hline & efficiency, \% & 95 & 95 & 95 & 92 & 95 \\
\hline & $f_{\mathrm{l}}, \mathrm{GHz}$ & 1.75 & 1.94 & 1.69 & 1.15 & 2.04 \\
\hline \multirow[t]{5}{*}{$H$-plane } & $f_{\mathrm{h}}, \mathrm{GHz}$ & 4.33 & 5.27 & 5.65 & 3.50 & 3.06 \\
\hline & $S_{11}, d B$ & -16.92 & -16.03 & -12.30 & -13.86 & -17.68 \\
\hline & gain, $\mathrm{dBi}$ & 2.45 & 2.53 & 2.62 & 2.00 & 0.69 \\
\hline & directivity, $\mathrm{dBi}$ & 2.67 & 2.85 & 2.85 & 2.86 & 2.86 \\
\hline & efficiency, \% & 95 & 93 & 95 & 82 & 61 \\
\hline
\end{tabular}

monopole antenna at $2.45 \mathrm{GHz}$ was carried out for crumpling cases 1 and 2 in both crumpling directions as shown in Fig. 7. A good agreement is achieved between simulation and measurement results.

\section{Performance of AMC antenna under crumpling conditions}

Since the limitations imposed by the simulation program to build a crumpled AMC antenna structure due to the total thickness of the structure and the feeding requirements to keep enough distance between the crumpling point and feeding line, the performance of 

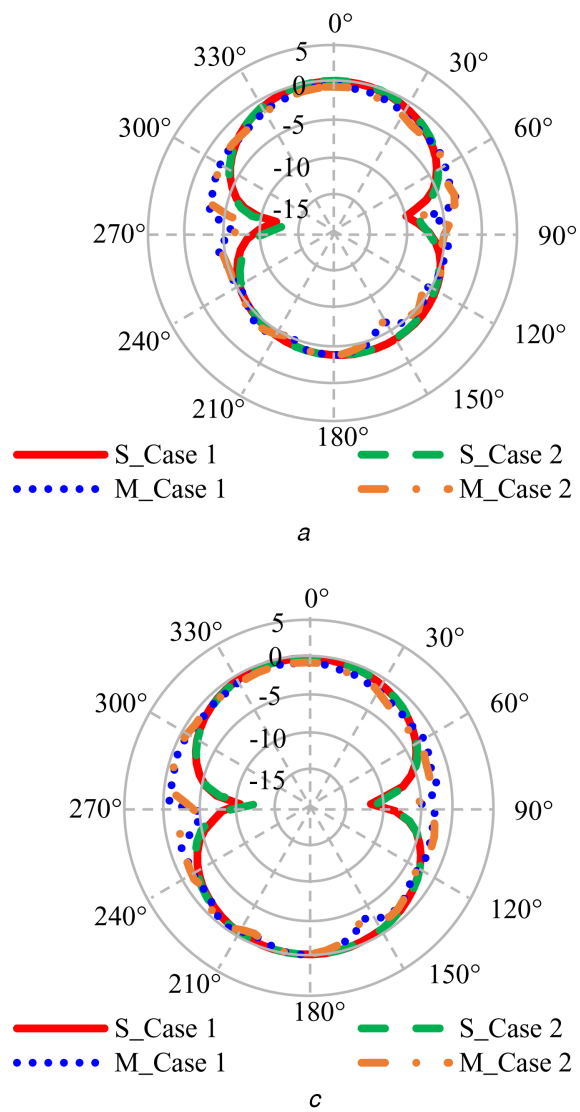

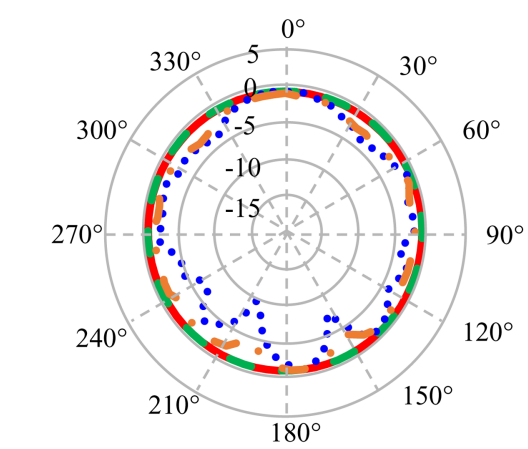

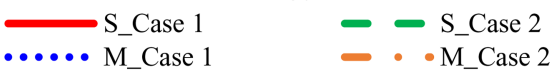

$b$

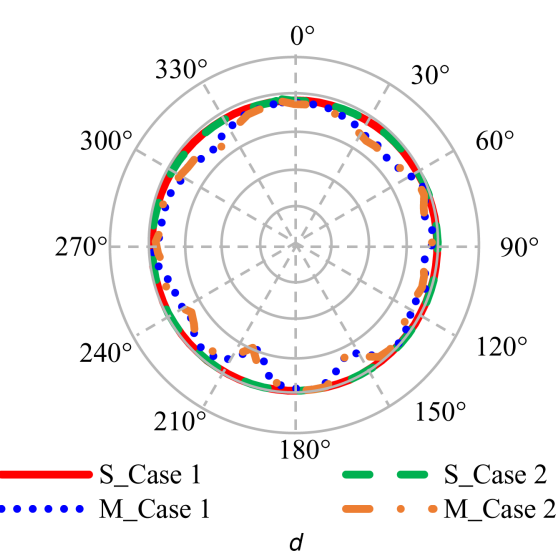

Fig. 7 Normalised radiation patterns at $2.45 \mathrm{GHz}$ based on simulation (S) and measurement (M) results for crumpled monopole antenna in (a) $E$-plane direction ( $E$-plane), (b) $E$-plane direction ( $H$-plane), (c) $H$-plane direction ( $E$-plane), (d) $H$-plane direction $(H$-plane)

Table 2 Dimensions of the crumpled AMC antenna

\begin{tabular}{lccc}
\hline & $M, \mathrm{~mm}$ & $\mathrm{~N}, \mathrm{~mm}$ & Antenna length, $\mathrm{mm}$ \\
\hline flat & - & - & 124.00 \\
series 1: case1 & 30 & 5 & 116.78 \\
case 2 & 30 & 6 & 114.25 \\
case 3 & 30 & 7 & 111.60 \\
series 2: case 1 & 31 & 5 & 117.48 \\
case 2 & 31 & 6 & 114.97 \\
case 3 & 31 & 7 & 111.89 \\
\hline
\end{tabular}

the AMC antenna under crumpling conditions has been studied only in the $E$-plane direction. The crumpling profile is defined in the same manner as for monopole antenna crumpling, where $(M)$ is the crumpling periodic length and $(N)$ is the crumpling depth.

\subsection{AMC antenna crumpling in E-plane}

A parametric study on the effects of change in crumpling profile parameters on the performance of the AMC antenna was carried out. AMC antenna crumpling was investigated for two series. Series 1 has three crumpling cases where $M$ is $30 \mathrm{~mm}$ and $N$ is 5, 6, and $7 \mathrm{~mm}$. In series $2, M$ is $31 \mathrm{~mm}$ while $N$ has the same values as in series 1. The dimensions of the AMC antennas for these crumpling series are shown in Table 2. The AMC antenna aperture is reduced due to crumpling from $124 \mathrm{~mm} \times 124 \mathrm{~mm}$ (flat) to a minimum value of $111.60 \mathrm{~mm} \times 124 \mathrm{~mm}$ obtained when $M=30$ $\mathrm{mm}$ and $N=7 \mathrm{~mm}$.

\subsection{Results and discussion of AMC antenna crumpling}

The reflection coefficients of the AMC antenna due to crumpling in series 1 and series 2 are depicted in Fig. 8. In general, crumpling the AMC antenna results in a shift in the resonance frequency and a reduction in the $-10 \mathrm{~dB}$ impedance bandwidth. The effect of changing $M$ value from 30 to $31 \mathrm{~mm}$ can be seen as a shift in the resonance frequency toward higher values. On the other hand, it can be concluded that changing the $N$ value has less impact on the AMC antenna performance. Crumpling in series 2 showed better matching within the frequency range of interest, $S_{11}$ being $<-10$ $\mathrm{dB}$, as compared with crumpling in series 1 . The $S_{11}$ measurement of the crumpled AMC antenna was carried out for case 2 of series 2 . It is worth mentioning that despite best efforts to fix the monopole antenna on the surface of the crumpled AMC structure, we noticed an air gap layer that might have caused discrepancies in the obtained results. In addition, the $S_{11}$ measurement of the crumpled AMC antenna in the $H$-plane is carried out for the same selected crumpling case, i.e. case 2 of series 2. Reflection coefficient results, as shown in Fig. $8 c$ showed that there are no significant changes compared with antenna crumpling in the $E$ plane.

The radiation characteristics of the AMC antenna at the resonance frequency of the antenna $\left(f_{\mathrm{r}}\right)$ and at $2.45 \mathrm{GHz}$ are summarised for crumpling conditions in series 1 and series 2 in Table 3. For the cases in which the AMC antenna experiences a multi-resonance behaviour, the resonance that is chosen for investigation is the closest to the ISM $2.45 \mathrm{GHz}$ band. The radiation characteristics of the AMC antenna for crumpling cases 1 and 2 in both series at $f_{\mathrm{r}}$ are in comparison with those obtained when the antenna is flat, while a degradation in the antenna performance is noticed due to the crumpling in case 3 with the $f_{\mathrm{r}}$ being shifted out of the ISM $2.45 \mathrm{GHz}$ band.

The most noticeable crumpling effect at $2.45 \mathrm{GHz}$ is a significant reduction in the antenna gain and efficiency of $6.69 \mathrm{~dB}$ and $41 \%$, respectively, due to crumpling in case 1 in series 1 . Please note that in this case, the resonance frequency is no longer at $2.45 \mathrm{GHz}$. Crumpling in case 2 in series 2, which represents the typical crumpling case that may take place, in reality, shows acceptable performance when compared with the antenna in the flat form.

To explain changes in AMC antenna radiation characteristics and input impedance matching due to crumpling conditions, the effect of AMC reflector crumpling on the $\pm 90^{\circ}$ reflection phase bandwidth was simulated and the results are summarised in 

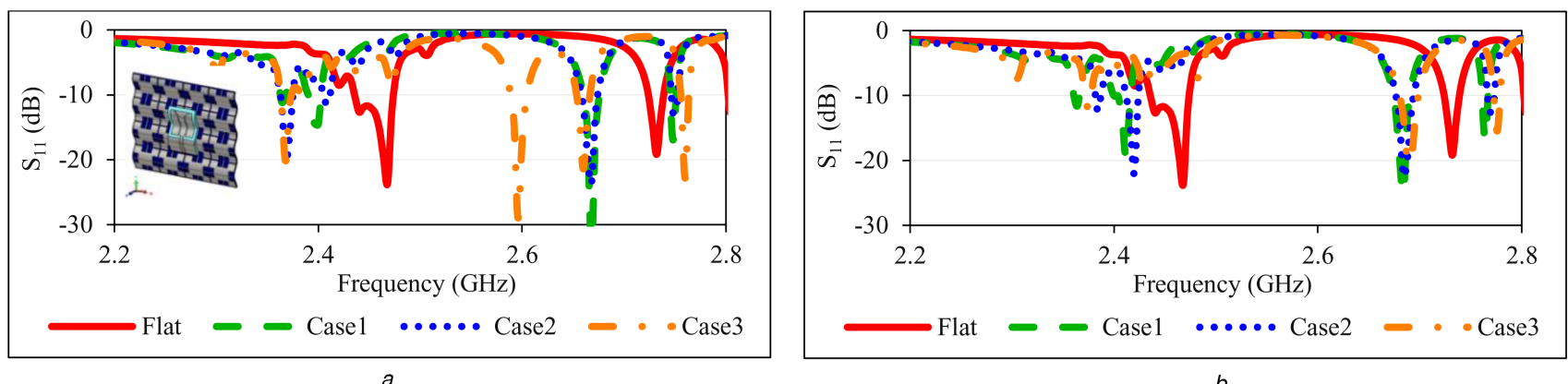

a

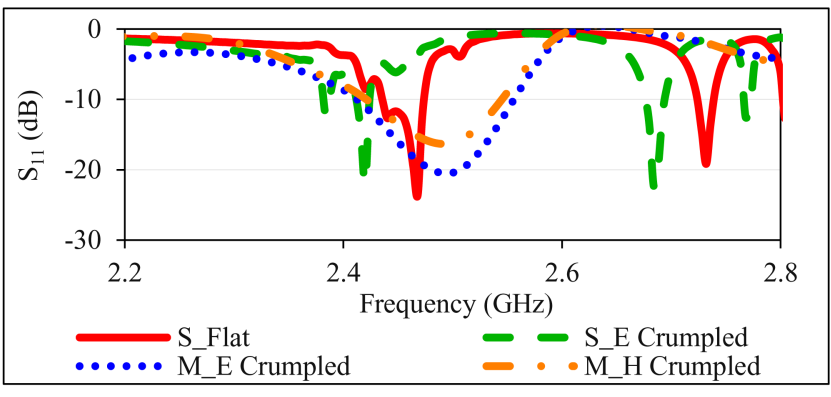

Fig. $8 S_{11}$ of crumpled AMC antenna

(a) Series 1: simulation results, (b) Series 2; simulation results, (c) Simulation $(S)$ and measurement $(M)$ results of $E$-plane crumpling and measurement $(M)$ results of $H$-plane crumpling in case 2 from series 2

Table 3 Radiation characteristics summary for AMC antenna crumpling in E-plane

\begin{tabular}{|c|c|c|c|c|c|}
\hline & Parameter & Flat AMC & Case 1 & Case 2 & Case 3 \\
\hline & $f_{\mathrm{r}}, \mathrm{GHz}$ & 2.46 & 2.40 & 2.41 & 2.36 \\
\hline \multirow[t]{9}{*}{ series 1} & gain at $f_{\mathrm{r}}, \mathrm{dBi}$ & 8.55 & 7.27 & 7.46 & 2.01 \\
\hline & directivity, $\mathrm{dBi}$ & 10.17 & 9.43 & 9.60 & 6.07 \\
\hline & efficiency, \% & 70 & 61 & 61 & 40 \\
\hline & FBR & 12.50 & 15.88 & 20.27 & 6.85 \\
\hline & gain at $2.45 \mathrm{GHz}, \mathrm{dBi}$ & 8.41 & 1.72 & 3.50 & 6.23 \\
\hline & directivity, $\mathrm{dBi}$ & 9.86 & 7.34 & 8.35 & 8.86 \\
\hline & efficiency, \% & 71 & 30 & 33 & 55 \\
\hline & FBR & 8.50 & 9.81 & 10.08 & 18.00 \\
\hline & $f_{\mathrm{r}}, \mathrm{GHz}$ & 2.46 & 2.41 & 2.41 & 2.70 \\
\hline \multirow[t]{8}{*}{ series 2} & gain at $f_{\mathrm{r}}, \mathrm{dBi}$ & 8.55 & 7.29 & 7.41 & 5.33 \\
\hline & directivity, $\mathrm{dBi}$ & 10.17 & 9.57 & 9.28 & 6.61 \\
\hline & efficiency, \% & 70 & 59 & 65 & 74 \\
\hline & FBR & 12.50 & 19.91 & 16.75 & 15.10 \\
\hline & gain at $2.45 \mathrm{GHz}, \mathrm{dBi}$ & 8.41 & 3.75 & 6.80 & 5.64 \\
\hline & directivity, $\mathrm{dBi}$ & 9.86 & 7.49 & 9.15 & 8.16 \\
\hline & efficiency, \% & 71 & 42 & 58 & 56 \\
\hline & FBR & 8.50 & 14.82 & 14.58 & 13.00 \\
\hline
\end{tabular}

Table 4 Zero reflection frequency point of crumpled AMC structure; frequencies are given in $(\mathrm{GHz})$

\begin{tabular}{lccc}
\hline & $f_{\mathrm{f}}^{*}$ & Zero reflection point & $f_{\mathrm{h}}{ }^{*}$ \\
\hline series 1: case 1 & 2.302 & 2.435 & 2.443 \\
case2 & 2.404 & 2.452 & 2.531 \\
case3 & 2.325 & 2.486 & 2.523 \\
series 2: case 1 & 2.365 & 2.456 & 2.514 \\
case 2 & 2.406 & 2.477 & 2.480 \\
case 3 & 2.406 & 2.500 & 2.701 \\
\hline
\end{tabular}

$f \mathrm{i}^{*}$ represents $+90^{\circ}$ phase point and $f \mathrm{~h}^{*}$ represents $-90^{\circ}$ phase point.

Table 4. It can be concluded that crumpling the AMC reflector shifts the point of zero phase reflection that represents the maximum constructive effect of antenna's image that can be achieved using the AMC reflector, which is neither at the resonance frequency of the crumpled AMC antennas nor at the $2.45 \mathrm{GHz}$ frequency point. However, one may expect that a significant improvement in antenna performance should be still achieved since these frequency points are within the $\pm 90^{\circ}$ reflection phase bandwidth for most of the studied crumpled AMC antennas. However, the achieved performance of the crumpled AMC antenna was not as good as that obtained when the AMC antenna is in the flat form.

\section{Performance of wearable antennas in proximity to human body models}

Two scenarios were examined for the assessment of the antenna performance on human body based on simulations carried out using CST MWS. Antennas were initially placed on a simplified three-layered planar rectangular human tissue model, in order to minimise the computational costs and simulation times. It was assumed that the curvature of the body can be neglected for 
Table 5 Electrical properties of the body tissues of the layered body model at $2.45 \mathrm{GHz}$ [18]

\begin{tabular}{|c|c|c|c|}
\hline Layer & Thickness, $\mathrm{mm}$ & Relative permittivity, F/m & Conductivity, S/m \\
\hline skin & 3 & 38.01 & 1.46400 \\
\hline fat & 7 & 5.28 & 0.10452 \\
\hline muscle & 60 & 52.73 & 1.73880 \\
\hline
\end{tabular}
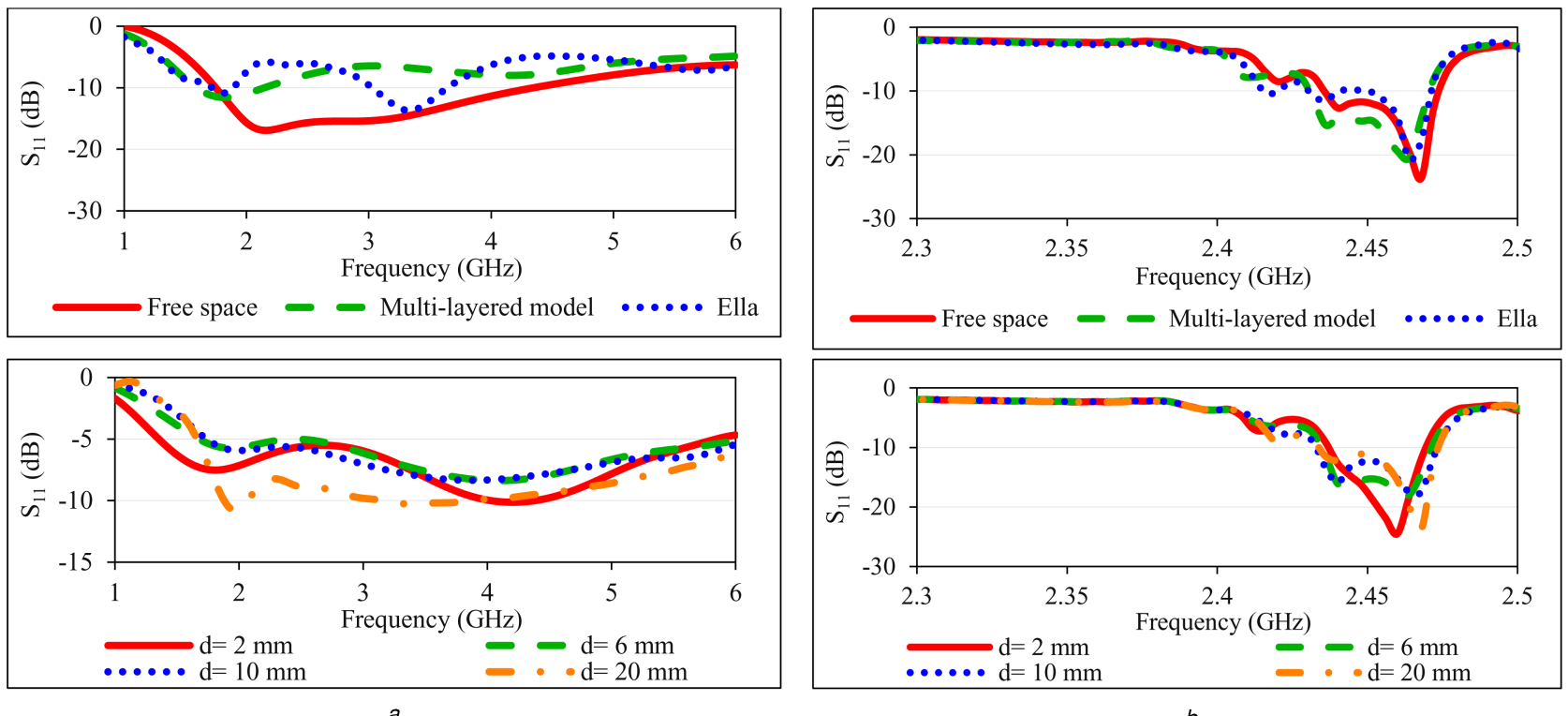

Fig. $9 S_{11}$ of wearable antennas; on different body models (top) and at different distances away from the multi-layered human body model (bottom) (a) Monopole antenna, (b) AMC antenna

physically small antennas. The simplified human body model consists of three tissue layers: skin, fat, and muscle, with an overall surface size of $200 \mathrm{~mm} \times 200 \mathrm{~mm}$, which is more than 3 times the surface area of the designed antenna. This layered body model represents most of the body regions quite well. Since fat tissue has similar properties to bone tissue and the electrical parameters of muscle tissue and many inner organs are similar [17]. The thickness and dielectric properties of the layers considered in the body model [18] are tabulated in Table 5 .

The antennas were subsequently simulated considering Ella model which represents a 26-year-old female with a height of 1.36 $\mathrm{m}$ and weight of $57.3 \mathrm{~kg}$ belonging to the virtual family [19]. A layer of Pellon fabric $\left(\varepsilon_{\mathrm{r}}=1.08, \tan \delta=0.008\right)$ of $3.6 \mathrm{~mm}$ thickness was placed between the antennas and the presenting body models during simulation in order to account for clothing.

Analysis of frequency detuning, antenna radiation characteristics, and SAR values was performed and summarised in the following subsections. Moreover, a parametric study on the antenna-body separation effect on the antennas' performance in terms of matching properties was carried out for general applicability to a wide range of operational scenarios.

\subsection{Frequency detuning}

Reflection coefficients of monopole and AMC antennas for freespace, multi-layered body model, and Ella body model conditions are shown in Fig. 9. In general, the highly dielectric nature of human tissue affects the matching properties of the antenna without AMC, causing both attenuation and detuning. A reduction of 79 and $75 \%$ in the $-10 \mathrm{~dB}$ impedance bandwidth in addition to the antenna not resonating within the ISM $2.45 \mathrm{GHz}$ band is observed when the conventional monopole antenna is placed close to the multi-layered body and Ella body models, respectively.

On the other hand, the AMC antenna retains its impedance matching for both scenarios. A small shift to a lower frequency of $2.464 \mathrm{GHz}$ is observed for the AMC antenna when placed close to human body models as compared with its resonance in free space at $2.468 \mathrm{GHz}$, making the antenna nearly $100 \%$ tolerant to the positioning on the lossy material, such as the human body.
Moreover, these performances confirm the validity of the choice and the effectiveness of the simplified multi-layered body model.

To consider the applicability of the AMC reflector on different wearable antenna operational scenarios, a parametric study on antenna-body separation distance $(d)$, using the simplified multilayered body model, was performed to study the stability of the antenna's matching properties. The distance between the antenna and tissue model (d) varied from 2 to $20 \mathrm{~mm}$. As shown in Fig. 9, monopole antenna resonance frequency was shifted when the antenna was brought closer to the surface of the tissue model. In addition, poor impedance matching within the ISM $2.45 \mathrm{GHz}$ band was observed. On the other hand, the AMC antenna shows steady impedance matching similar when it functions in free space.

\subsection{Radiation characteristics}

Table 6 summarises the radiation properties of monopole and AMC antennas evaluated when placed on human body models and the case of free space condition. Important observations can be made from the presented results. The first is that the presence of the human body deteriorates the gain and efficiency of the antenna when the AMC reflector is not integrated. The second observation is that the AMC reflector effectively isolates the human body model from the radiating antenna since the radiation characteristics in terms of gain and efficiency remain comparable in the presence and absence of the human body. The results of reflection coefficients in combination with the radiation characteristics of studied antennas demonstrate the necessity of the AMC reflector in order for the antenna to be effectively and efficiently used for wearable applications since it makes the antenna less affected by the human body. Moreover, one can appreciate that despite larger discrepancies are found when focusing on antennas radiation performances considering the simplified multi-layered and Ella body models in comparison with matching properties, the simplified multi-layered body model is a satisfactory representation of the human body. 
Table 6 Radiation characteristics summary for wearable antennas on human body models at $2.45 \mathrm{GHz}$

\begin{tabular}{lccc}
\hline & Environment & Gain, dBi & Efficiency, \% \\
\hline monopole antenna & free space & 2.45 & 95 \\
& multi-layered model & -1.69 & 10 \\
AMC antenna & Ella model & 0.35 & 21 \\
& free space & 8.41 & 71 \\
& multi-layered model & 8.40 & 66 \\
\hline
\end{tabular}

Table 7 SAR analysis summary of wearable antennas at $2.45 \mathrm{GHz}$

\begin{tabular}{|c|c|c|c|c|c|c|}
\hline & $1 \mathrm{~g}$ tissue, $\mathrm{W} / \mathrm{kg}$ & $10 \mathrm{~g}$ tissue, $\mathrm{W} / \mathrm{kg}$ & Point SAR, W & $\mathrm{HPBW}$ in E-plane, ${ }^{\circ}$ & HPBW in $H$-plane, ${ }^{\circ}$ & FBR \\
\hline flat monopole & 16.46 & 9.390 & 56.62 & 61.5 & 81.2 & 25.52 \\
\hline crumpled monopole 1 & 11.71 & 6.704 & 40.49 & 57.5 & 80.3 & 30.61 \\
\hline crumpled monopole 2 & 17.50 & 9.825 & 61.13 & 58.6 & 80.9 & 28.66 \\
\hline flat AMC & 0.33 & 0.166 & 2.14 & 66.9 & 45.5 & 32.33 \\
\hline crumpled AMC & 0.19 & 0.098 & 0.75 & 60.6 & 40.3 & 35.18 \\
\hline
\end{tabular}

\subsection{Analysis of SAR}

SAR analysis is essential to evaluate the performance of the antenna when it is close to the human body and to consider the safety concerns and limits imposed by the standards. The maximum SAR limit is $1.6 \mathrm{~W} / \mathrm{kg}$ for any $1 \mathrm{~g}$ tissue by the Institute of Electrical and Electronics Engineers standard [20] or $2 \mathrm{~W} / \mathrm{kg}$ for any $10 \mathrm{~g}$ tissue by the International Commission on Non-Ionizing Radiation Protection (ICNIRP) standard [21]. SAR is a measure of the rate at which energy is absorbed by the human body when exposed to an electromagnetic field. The mass-averaged SAR method (typically 1 and $10 \mathrm{~g}$ ), using the simplified multi-layered body model, was used in the simulation for $1 \mathrm{~W}$ delivered power.

SAR analysis has been carried out for monopole and AMC antennas in the flat form and under crumpling conditions of $N=6$ $\mathrm{mm}$ and $M=31 \mathrm{~mm}$. This crumpling case has been chosen for SAR analysis since it is the common crumpling case among aforementioned crumpling cases of monopole and AMC antennas, which makes it easy for SAR comparison. Simulated averaged SAR values are summarised in Table 7, where 'crumpled monopole 1' and 'crumpled monopole 2' are monopole antennas crumpled in $E$ - and $H$-plane, respectively, and 'crumpled AMC' is the AMC antenna crumpled in the $E$-plane.

The mass-averaged method depends on the surface area of the human body model chosen for investigation; however, this method is still valid to show the benefit of using AMC reflectors in wearable antenna design. Maximum point SAR analysis was performed, and the results are shown in Table 10. In addition, half power beam width (HPBW) and FBR values are summarised and listed in Table 10 in order to study the effects of variations in radiation characteristics on the obtained SAR.

For the considered input power, a significant reduction in SAR at $2.45 \mathrm{GHz}$ is achieved using an AMC reflector for the antenna in the flat form and under crumpling conditions. The obtained SAR levels of the AMC antenna are within the specified limits allowed by the aforementioned standards, which give a reliable indication of the benefits of AMC being used as an antenna ground plane. In addition, antenna crumpling showed variations in the obtained SAR compared with the flat form. Crumpling antennas in the $E$ plane result in a lower SAR values compared with SAR values of antennas in the flat form. On the other hand, monopole crumpling in the $H$-plane results in a higher SAR levels compared with SAR levels of the flat antenna.

\section{Conclusion}

In this study, we presented a textile-based monopole antenna design integrated with a flexible AMC ground plane for wearable applications. The in-phase reflection characteristic of the AMC structure significantly enhanced the radiation characteristics of the antenna in terms of antenna gain and front to back ratios. Furthermore, it reduced the undesired electromagnetic radiation towards the human body, which is essential to the performance of wearable antenna systems. The calculated SAR values for the AMC-based antenna were very low, with a reduction of more than $90 \%$ compared with the SAR values of the same antenna without an AMC reflector. Moreover, flexibility tests in terms of antennas' performance under crumpling conditions were carried out to see the effect of shifting the zero in-phase point from that of the flat AMC reflector. It was concluded that AMC antennas are more susceptible to crumpling but provides better protection from radiation towards the body.

\section{References}

[1] Osman, M.A.R., Abd Rahim, M.K., Samsuri, N.A., et al.: 'Embroidered fully textile wearable antenna for medical monitoring applications', Prog. Electromagn. Res., 2011, 117, pp. 321-337

[2] Zhu, S., Langley, R. 'Dual-band wearable textile antenna on an EBG substrate', IEEE Trans. Antennas Propag., 2009, 57, (4), pp. 926-935

[3] Klemm, M., Troester, G.: 'Textile UWB antennas for wireless body area networks', IEEE Trans. Antennas Propag., 2006, 54, (11), pp. 3192-3197

[4] Rais, N.H.M., Soh, P.J., Malek, F., et al.: 'A review of wearable antenna'. Proc. Loughborough Antennas and Propagation Conf. (LAPC), Loughborough, UK, 16-17 November 2009, pp. 225-228

[5] Velan, S., Sundarsingh, E., Kanagasabai, M., et al.: 'Dual-band EBG integrated monopole antenna deploying fractal geometry for wearable applications', IEEE Antennas Wirel. Propag. Lett., 2014, 14, pp. 249-252

[6] Rosen, A., Stuchly, M., Vander Vorst, A.: 'Applications of RF/microwaves in medicine', IEEE Trans. Microw. Theory Tech., 2002, 50, (3), pp. 963-974

[7] Raad, H.R., Abbosh, A.I., Al-Rizzo, H.M., et al.: 'Flexible and compact AMC based antenna for telemedicine applications', IEEE Trans. Antennas Propag., 2013, 61, (2), pp. 524-531

[8] Kim, S., Ren, Y.-J., Lee, H., et al.: 'Monopole antenna with inkjet-printed EBG array on paper substrate for wearable applications', IEEE Antennas Wirel. Propag. Lett., 2012, 11, pp. 663-666

[9] Alemaryeen, A., Noghanian, S.: 'AMC integrated textile monopole antenna for wearable applications', $A C E S J ., 2016,31$, (6), pp. 612-618

[10] Bai, Q., Langley, R.: 'Crumpling of PIFA textile antenna', IEEE Trans. Antennas Propag., 2012, 60, (1), pp. 63-70

[11] Bai, Q., Langley, R.: 'Crumpled integrated AMC antenna', Electron. Lett., 2009, 45, (13), pp. 662-663

[12] Bai, Q., Langley, R.: 'Crumpling of compact textile antennas'. Proc. European Conf. on Antennas \& Propagation (EuCAP), April 2011, pp. 36313634

[13] CST Microwave Studio version $2015 \mathrm{http} /$ www.cst.com

[14] Mantash, M., Tarot, A.-C., Collardey, S., et al.: 'Investigation of flexible textile antennas and AMC reflectors', Int. J. Antennas Propag., 2012, 2012, pp. $1-10$

[15] Alemaryeen, A., Noghanian, S., Fazel-Rezai, R.: 'EBG integrated textile monopole antenna for space health monitoring application'. Proc. IEEE AP-S Int. Symp, Canada, July 2015, pp. 1209-1210

[16] Yang, F., Rahmat-Samii, Y.: 'Reflection phase characterizations of the EBG ground plane for low profile wire antenna applications', IEEE Trans. Antennas Propag., 2003, 51, (10), pp. 2691-2703

[17] Klemm, M., Troester, G.: 'EM energy absorption in the human body tissues due to UWB antennas', Prog. Electromagn. Res., 2006, 62, pp. 180-261

[18] Gemio, J., Parron, J., Soler, J.: 'Human body effects on implantable antennas for ISM bands applications: models comparison and propagation losses study', Prog. Electromagn. Res., 2010, 110, pp. 437-452

[19] Gosselin, M., Neufeld, E., Moser, H., et al: 'Development of a new generation of high-resolution anatomical models for medical device evaluation: the virtual population 3.0', Phys. Med. Biol., 2014, 59, (18), pp. $5287-5303$ 
[20] IEEE Std C95.3-2002: 'IEEE recommended practice for measurements and computations of radio frequency electromagnetic fields with respect to human exposure to such fields, $100 \mathrm{kHz}-300 \mathrm{GHz}, 2002$
[21] Commission of European Communities: 'Council recommendation on limits for exposure of the federal public to electromagnetic fields: $0 \mathrm{~Hz}-300 \mathrm{GHz}$ (June 1998) 